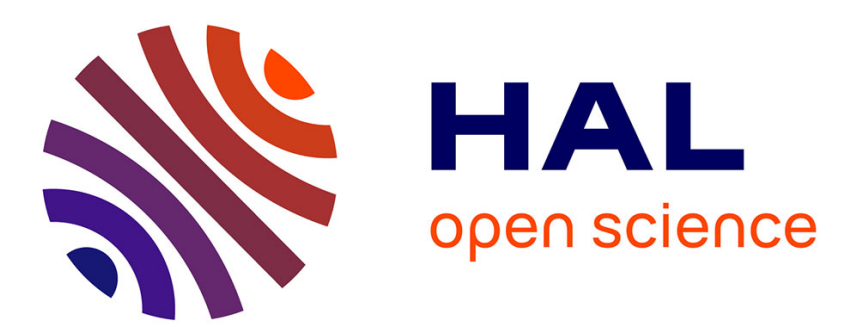

\title{
Augmented Reality for People with Visual Impairments: Designing and Creating Audio-Tactile Content from Existing Objects
}

Lauren Thévin, Anke Brock

\section{- To cite this version: \\ Lauren Thévin, Anke Brock. Augmented Reality for People with Visual Impairments: Designing and Creating Audio-Tactile Content from Existing Objects. ICCHP'18 16th International Conference on Computers Helping People with Special Needs, Jul 2018, Linz, Austria. hal-01769602}

\section{HAL Id: hal-01769602 \\ https://hal.science/hal-01769602}

Submitted on 20 Aug 2018

HAL is a multi-disciplinary open access archive for the deposit and dissemination of scientific research documents, whether they are published or not. The documents may come from teaching and research institutions in France or abroad, or from public or private research centers.
L'archive ouverte pluridisciplinaire HAL, est destinée au dépôt et à la diffusion de documents scientifiques de niveau recherche, publiés ou non, émanant des établissements d'enseignement et de recherche français ou étrangers, des laboratoires publics ou privés. 


\title{
Augmented Reality for People with Visual Impairments: Designing and Creating Audio-Tactile Content from Existing Objects
}

\author{
Lauren Thevin ${ }^{1}$ and Anke M. Brock ${ }^{2}$ \\ ${ }^{1}$ Inria Bordeaux, France \\ ${ }^{2}$ ENAC - University Toulouse, France \\ lauren.thevin@inria.fr; anke.brock@enac.fr
}

\begin{abstract}
Tactile maps and diagrams are widely used as accessible graphical media for people with visual impairments, in particular in the context of education. They can be made interactive by augmenting them with audio feedback. It is however complicated to create audiotactile graphics that have rich and realistic tactile textures. To overcome these limitations, we propose a new augmented reality approach allowing novices to easily and quickly augment real objects with audio feedback. In our user study, six teachers created their own audio-augmentation of objects, such as a botanical atlas, within 30 minutes or less. Teachers found the tool easy to use and were confident about re-using it. The resulting augmented objects allow two modes: exploration mode provides feedback on demand about an element, while quiz mode provides questions and answers. We evaluated the resulting audio-tactile material with five visually impaired children. Participants found the resulting interactive graphics exciting to use independently of their mental imagery skills.
\end{abstract}

\section{Introduction}

In special education environments, tactile maps and graphics are widely used, for example in O\&M (Orientation \& Mobility) classes, or for mathematics and biology. There are two design methods for augmenting tactile media with audio feedback. First, tactile graphics can be hand-made, e.g. using paper, tissue or real objects [1] so that they possess a rich tactile dimension (e.g. texture, thermal properties). Yet, it is difficult to make those graphics interactive. Second, the tactile mediums can be digitally designed and edited, then printed and easily made interactive using Text-to-Speech synthesis (TTS) or Braille displays [2]. But such objects are limited, for instance to swellpaper or 3D print. In specialized schools, many objects for visually impaired (VI) people are hand-made, such as small-scale models or students' works made in class. At the same time, people who want to augment tactile objects with audio feedback (e.g. teachers), do not necessarily master $2 \mathrm{D}$ and $3 \mathrm{D}$ modeling techniques. It is likely that they have knowledge about office software applications, but no specialized computer skills. 


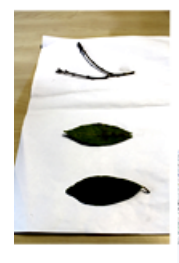

(a)

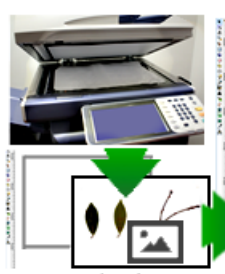

(b)

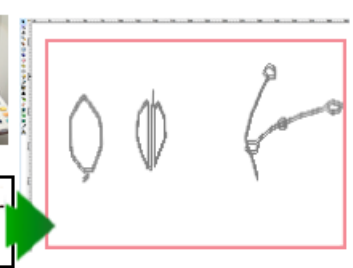

(c)

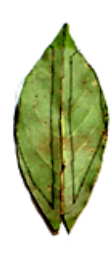

(d)

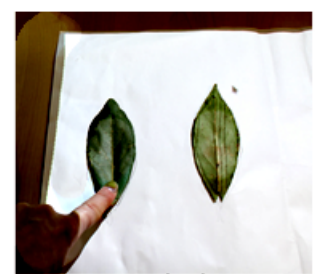

(e)

Fig. 1. Augmentation process: (a) designing the tactile content with real objects, (b) scanning it and using the resulting image as basis for (c) creating the SVG file with interactive zones, using PapARt (Fig. 2, left) to project this image on a table, (d) superimposing the projected interactive zones and the real objects, and (e) using the object as an audio-tactile interactive medium.

We propose a first approach of creating augmented content for Augmented Reality (AR) with real objects, thus enabling both rich tactile feedback and interactivity. The audio-content is associated with a SVG file (vector format often used for designing raised-line maps [2]). We present the necessary steps in Figure 1. We illustrate our work with two use cases: a botanical atlas and a geographic map. This approach allows teachers to design their own pedagogical audio-tactile content from existing objects (leaves, branches, small-scale models, etc.). For the resulting audio-tactile medium, two modes of use are proposed : exploration mode with audio feedback on demand, and quiz mode. The augmented prototype was designed and evaluated in collaboration with IRSA (special education school in Bordeaux). We tested the creation of audio-tactile content with teachers, and the usability of the resulting audio-tactile graphics with VI students.

\section{State of the Art}

\subsection{Tactile and Audio Modalities in Interactive Graphics}

Tactile graphics serve the same use cases as pictures for sighted people: they are small-scale representations used e.g. for education. Audio feedback allows to create interactive tactile graphics, using for instance TTS to describe content and events [3]. The tactile modality encodes various types of information, and supports the creation of a rich mental imagery also for VI people [4]. Tactile maps, interactive or not, can be created in relief with a single material (e.g. using laser cutting, swell-paper [5], 3D printing [6]), or composed from objects with various textures [7]. Tactile graphics can support several haptic sensations: graining, softness/stiffness, shapes (circle/square, full/hollow), relief (cavity/bump), thermal behavior (cold/warm), etc. [8]. Using various textures helps to distinguish tactile elements. Using realistic textures can help to recognize elements in real situations. In our use case of a botanical atlas, some plants have a particular texture (such as succulent plants) that makes them easy to recognize by touch. 
Braille text normally accompanies tactile graphics [5]. As an alternative, audio feedback allows to augment objects [3]. Unlike with Braille text, there is no need for moving the hands between the object and the associated caption [2]. The exploration and use of audio-tactile maps has proved more effective for complex content [7] than the use of Braille maps, and more efficient and satisfying for simple content [2]. Moreover, interactivity makes it possible to provide adaptive feedback depending on the context for the same tactile support: generic captions for superficial exploration, detailed descriptions for precise exploration, and an interactive answer-question system for testing knowledge [9].

\subsection{Augmentation Process}

We are interested in augmenting objects and making them interactive using AR. $\mathrm{AR}$ is not limited to visual modalities, but is defined as the combination of real objects with virtual objects in real time within a real environment independently of the modalities (vision, audition, touch,...) [10]. In our case, physical objects are combined with virtual audio feedback. We observe two main processes used to augment real objects with digital content. The first approach is to augment the object itself with electrical components [7] or to integrate components directly inside the object material [11]. But this approach offers little flexibility for the augmented content as it is integrated in the hardware. The second approach is to integrate augmented content in the digital representation of the object, e.g. vector images printed later as a raised-line map [9]. This object is then made physical, e.g. with 3D printing. Modeling objects with the associated digital content allows to attach several annotations to the same object (e.g. content with increasing detail). The physical object can then be linked with the digital object and its augmented content. A tracking system can follow the object and the users action [12]. However in this approach, the augmented object is either artificially created or digitally modeled based on an existing object.

To sum up, creating audio-tactile media is possible with real objects as a starting point or respectively by modeling and annotating digital objects. Yet, these two approaches require advanced skills in computer sciences and for building specific hardware. As far as we know, even for the tools made and tested
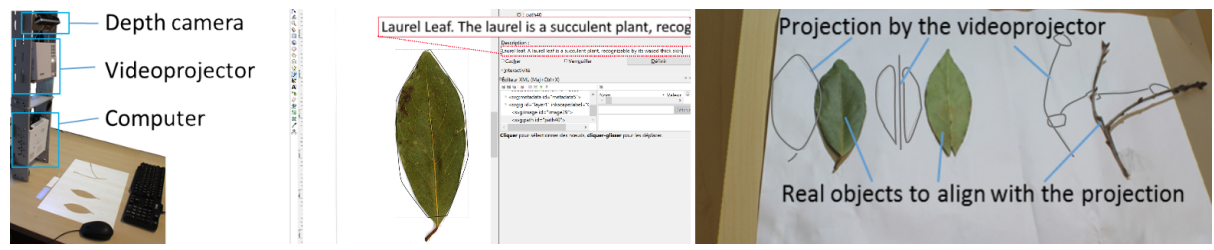

Fig. 2. Left: PapARt Hardware. Middle: SVG file with drawing over the scanned image. An audio-description is associated with the drawn elements. Right: Overlaying process. The projection displays the interactive zones. Once the tactile medium is aligned with the projection, the audio-tactile content is ready to use (here projection and objects are not aligned for clarity of image). 
in participatory design approaches, such as [9], the audio-tactile supports were developed by the researchers and not the target users (e.g. teachers).

\section{Proposed System}

Our audio-tactile AR system should be compatible with the tactile contents usually used by teachers (swellpaper, 3D printing, scratch, small-scale models), and their design should be possible for people with no specific computer skills.

\subsection{Hardware}

The technical system is composed of the existing PapARt framework (software and hardware) [13], a PC for the SVG file creation, a scanner, and a sheet of paper with tactile elements placed on it (see Fig. 2). PapARt uses a depth camera to trigger the interactive zones usually on a table surface based on the hand position (the arm is detected, then the hand and fingers position). The contact of the finger with the tactile map is detected using depth analysis.

\subsection{Creation of Interactive Content}

Defining the Content. To create the tactile content, real objects are glued to an A3/A4 paper sheet which serves as support (see Fig. 1).

Defining the Reference Frame to Superimpose Annotations. One of the main issues in AR is to link an object with its augmented content. We propose to scan a A3/A4 paper with the tactile content. The scanned image becomes a background in a vector graphics software (Inkscape) to draw shapes directly on top of it, respecting de facto the scale and alignment with the real graphic. the shapes in SVG format have a textual description rendered by TTS when using the interactive prototype and additional XML fields. The users can add the fields "step" and "question" to any shape to create a quiz (see below).

Usage. When PapARt hardware and software are launched, the user chooses the correct SVG image in the application. The image is then projected, and the projection overlaid on the physical support. Thus, the map or diagram becomes audio-tactile and usable in two modes: 1) in exploration mode an audiodescription is provided once the objects are pointed at as in [9], and 2) in quiz mode the system reads questions which the user solves by pointing to the requested element. If the pointed object is not correct, the computer provides directional help to redirect the finger as described in [9]. It is possible to load another SVG file for the same tactile content so the audio-content can evolve in the same session (e.g. quiz getting progressively more difficult).

\section{Evaluation}

Six O\&M Instructors and teachers were asked to evaluate the creation process, and five VI students were asked to evaluate the resulting audio-tactile media. We 

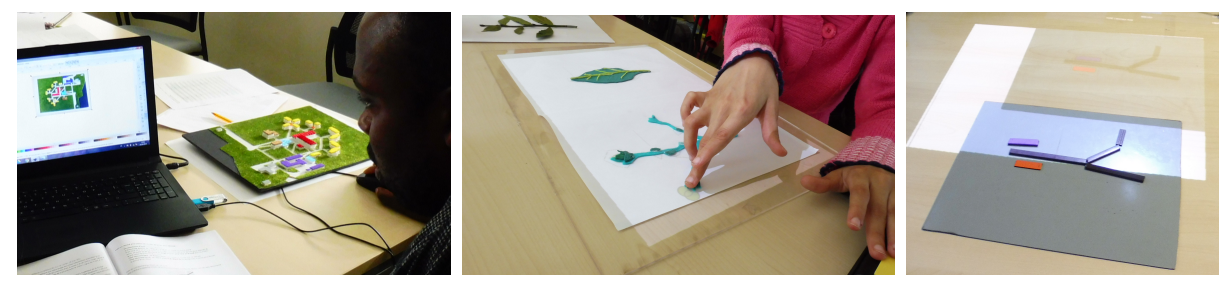

Fig. 3. Left: User Test 1, augmentation of a map and botanical atlas. Middle: User Test 2, two botanical atlases visible: one with felt and baize, one with real leaves on the background. Right: proof of concept: augmentation of a magnetic board

also evaluated a proof of concept. The user tests were approved by the ethical committee COERLE at Inria.

User Test 1: Can people without Specific Computer Skills Annotate Existing Tactile Material with Audio Feedback? Three O\&M instructors from IRSA were asked to augment an existing tactile map of the school. Three different teachers (2 from IRSA: biology, tutor; 1 teacher in a primary school) were asked to create and augment a tactile biological atlas. We assessed the workload using Nasa-TLX [14] and the usability using UEQ [15]. To be sure that the system is compatible with the available resources and the usual activity of the instructors and teachers, we first asked which material is available at their workplace. We asked the users to spontaneously design a tactile support and to describe the audio feedback which the support should have. This allowed to verify that our audio augmentation process is consistent with the audio interaction which teachers expect. To be sure the users were able to create the audio augmentation autonomously, we provided a manual and asked the participants to follow the instructions.

User Test 2: Are the Created Audio-Tactile Supports Acceptable and Usable by Students with VI? We tested whether the created maps are usable by the end users (i.e. VI children). Five VI children (11 to 13 years old, 2F and 3M, 2 low vision and 3 blind) were asked to use the audio-tactile supports created in User Test 1 by one of the previously participating teachers (map and botanical atlas). The children's teacher was present during the experimentation. We assessed the usability with the UEQ [15].

Proof of Concept: Are Existing Tactile Supports Easy to Annotate? We validated that the proposed design process provides the possibility to augment various existing tactile supports which are not specifically designed to be audio-augmented. Three existing tactile supports were augmented by the research team with audio feedback as requested by instructors of IRSA.

\subsection{Results}

User Test 1. All users succeeded in creating the audio-tactile content. The content for exploration mode was intuitive and easy for all, while the creation of quiz content was perceived as complicated by some users even if they succeeded. 

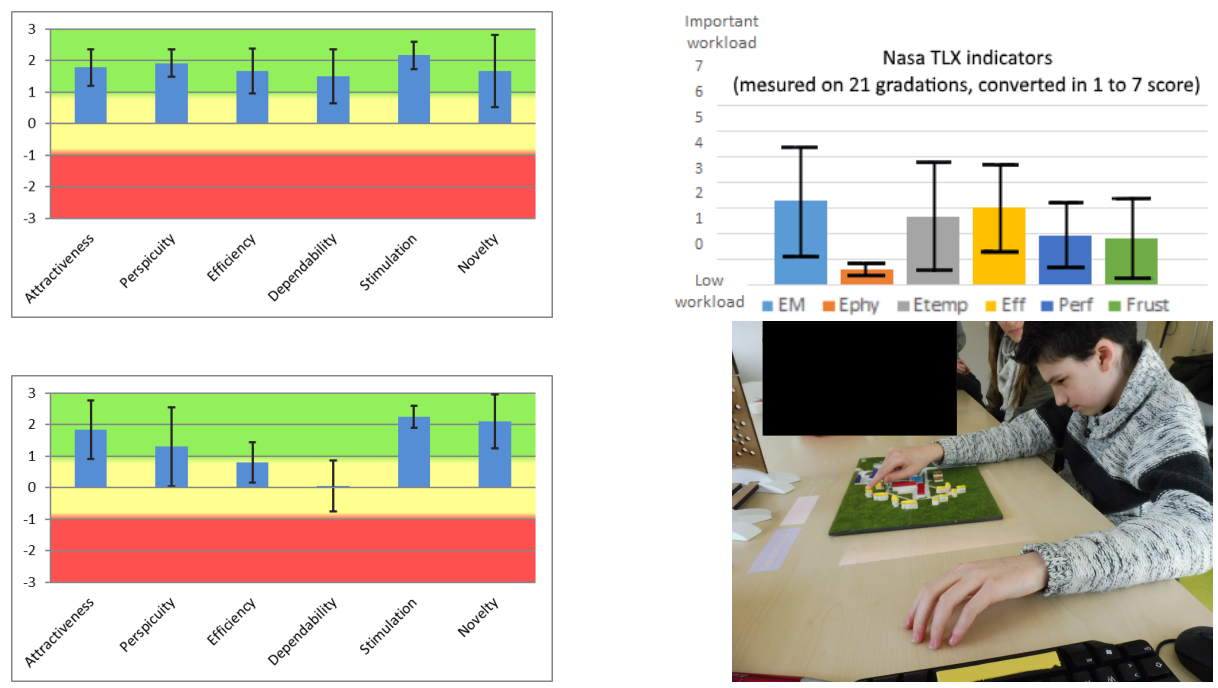

Fig. 4. TOP. Left Results of UEQ for User Test 1: all criteria are evaluated as good or very good. Right: Results of Nasa-TLX for User Test 1: none of the average indicators is associated with important workload. BOTTOM. Left Results of UEQ for User Test 2: lower scores for efficiency and dependability can be explained by finger detection. Right A visually impaired child exploring the map.

The average time for describing the tactile support was $11 \min (\min : 2$, max: 23), $13 \min (\min : 8$, max: 25) for creating the tactile support, $4 \min (\min : 1$, max: 10) for describing the audio-augmentation and $27 \min (\min : 15$, max: 51) for creating the audio augmentation. All participants needed one more iteration to adjust the audio feedback. The main errors in creating audio feedback were: confusion between questions that can be answered by pointing at an element (e.g. where is the library?) and questions that require verbal replies which is not foreseen in the system (e.g. which building is here?); and adjustment if the answering zone was first too small. Because a scanner was not always available, some of the augmentations were done using a photo, taken as vertical as possible. The results of Nasa-TLX and UEQ (Fig. 4) show that the system is usable without knowledge in programming and vector drawing. A participant noticed that "It is complicated the first time, because I never used it [Bezier curves, inkscape]. But now I feel I can do it again, and it will be ok. I need to manipulate only one software." One user was missing labels indicating which parts of the leaves or map elements were interactive. Such "interactive points" can be made with our system without electronic devices on the tactile maps. Some negative remarks concerned Inkscape (not usable with a picture that has a large filesize as it become slow, no feedback regarding actions).

User Test 2. In this user test we tested the audio-tactile document designed by the professors with VI students. The main result, noticed also by the teachers present during the experiment, was that the technology is interesting for chil- 
dren with good mental representation skills who are already attracted by tactile images ( 3 of 5 participants), as well as for the children for whom mental imagery tasks are more challenging and who do not necessarily appreciate tactile images ( 2 of 5 participants). Indeed, 4 children want to participate in future sessions with our prototype; and 3 wanted to continue exploring new tactile supports after the experiment ended. Even though the system is usable by both low vision and blind people, the feedback about the detection of finger positions is entirely visual and thus not accessible to blind people. Moreover, concepts as "no finger should hide other fingers" (to avoid occlusion for the image recognition) are hard to explain. Yet, such feedback can be useful as sometimes the finger detection for the younger participants was not very precise. We think this could be fixed with a better calibration of the system.

Proof of Concept The instructors from IRSA provided 3 existing tactile supports which we augmented. Students had built a tram station with magnets on a magnet board (Fig. 3). The time to augment was $6 \mathrm{~min}$, and it was fully operational after 1 additional iteration (adapting the text read by the TTS). One usage was identified: by projecting a "photo" of the original magnets' positions, it is possible to re-create the work of the students later on. The Velcro board had similar results. The small-scale model of a house had cardboard as vertical walls and textures on the floor. The time for augmentation of this model was $15 \mathrm{~min}$, the audio-content was fully operational after 1 more iteration (adapting the interactive zones with the finger detection due to the 3D structure). We also built a quiz mode where a question could have multiple solutions.

\section{Discussion \& Perspectives}

In this paper we presented the use of the PapARt AR toolkit [13] and Inkscape to easily and quickly make tactile material interactive. We tested the creation process with teachers. Generally, teachers found the tool easy to use and were confident about re-using it. Then, we evaluated the created material with VI children. They found it exiting and were willing to use it again. However, the finger detection needs to be improved. The annotation of real objects presented in this paper opens new perspectives, such as creating tactile and tangible prototypes in creativity sessions based on manual activities with physical materials (lego, playdough etc.), which can become interactive within 30min. Some negative feedback concerned the usability of inkscape. Therefore we propose that in the future, the interactive content could be directly created using PapARt only.

Acknowledgment. We thank the participants (teachers and students) especially C. Briant; and J. Laviole (Reality Tech) and J. Albouys for participating in the design of this tool. Done with the support of the EU Erasmus+ Pr. no 2016-1-EL01-KA201-023731. 


\section{References}

1. P. Edman. Tactile graphics. American Fnd. for the Blind, NY, USA, may 1992.

2. Anke M. Brock, Philippe Truillet, Bernard Oriola, Delphine Picard, and Christophe Jouffrais. Interactivity Improves Usability of Geographic Maps for Visually Impaired People. Human-Computer Interaction, 30(2):156-194, 2015.

3. J. Ducasse, A. Brock, and C. Jouffrais. Accessible interactive maps for visually impaired users. In Mobility of Visually Impaired People, pages 537-584. Springer, 2018.doi: 10.1145/2505401.2505402.

4. Z. Cattaneo and T. Vecchi. Blind vision: the neuroscience of visual impairment. MIT Press, 2011.doi: https://doi.org/10.7551/mitpress/9780262015035.001.0001.

5. A.F. Tatham. The design of tactile maps: theoretical and practical considerations. In K Rybaczak and M Blakemore, editors, Proceedings of international cartographic association: mapping the nations, pages 157-166, London, UK, 1991.doi: 10.1145/2982142.2982163. ICA.

6. T. Gotzelmann. LucentMaps 3D Printed Audiovisual Tactile Maps for Blind and Visually Impaired People. The 18th ACM Conf. ASSETS '16, pages 81-90, 2016.

7. S. Giraud, A. M. Brock, M. J-M. Macé, and C. Jouffrais. Map learning with a $3 \mathrm{~d}$ printed interactive small-scale model: Improvement of space and text memorization in visually impaired students. Frontiers in psychology, 8:930, 2017.doi: 10.3389/fpsyg.2017.00930.

8. R. L. Klatzky and S. J. Lederman. Touch. Handbook of psychology, 2003.

9. J. Albouys-Perrois, J. Laviole, C. Briant, and A. Brock. Towards a Multisensory Augmented Reality Map for Blind and Low Vision People: a Participatory Design Approach. International Conference CHI 2018, 2018.

10. R. Azuma, R. Behringer, S. Feiner, S. Julier, and B. Macintyre. Recent Advances in Augmented Reality. IEEE CGESA, 2011(December):1-27, 2001.doi: $10.1109 / 38.963459$.

11. C. Daudén Roquet, J. Kim, and T. Yeh. 3D Folded PrintGami: Transforming Passive 3D Printed Objects to Interactive by Inserted Paper Origami Circuits. DIS16, pages 187-191, 2016.doi: 10.1145/2901790.2901891.

12. L. Shi, Y. Zhao, and S. Azenkot. Markit and Talkit: A Low-Barrier Toolkit to Augment 3D Printed Models with Audio Annotations. In Proceedings of the 30th Annual Symposium UIST '17, pages 493-506, 2017.doi:10.1145/3126594.3126650.

13. J. Laviole and M. Hachet. PapARt: Interactive 3D graphics and multi-touch augmented paper for artistic creation. IEEE 3DUI'12 - Proceedings, pages 3-6, 2012. doi:10.1109/3DUI.2012.6184167.

14. Sandra G. Hart. Development of NASA-TLX (Task Load Index): Results of Empirical and Theoretical Research. In P. A. Hancock and N. Meshkati, editors, Human Mental Workload, volume 52 of Advances in Psychology, chapter 12, pages 139-183. Elsevier, advances i edition, 1988.doi:10.1016/S0166-4115(08)62386-9.

15. Bettina Laugwitz, Theo Held, and Martin Schrepp. Construction and Evaluation of a User Experience Questionnaire. In Andreas Holzinger, editor, HCI and Usability for Education and Work, volume 5298 of Lecture Notes in Computer Science, pages 63-76. Springer Berlin Heidelberg, Berlin, Heidelberg, 2008.doi:10.1007/978-3-54089350-9. 